\title{
Dynamics of Populations of Integrate-and-Fire Neurons, Partial Synchronization and Memory
}

\author{
Marius Usher \\ Computation and Neural Systems 216-76, Caltech, Pasadena, CA 91125 USA \\ Heinz Georg Schuster \\ Institut für Theoretische Physik, Universität Kiel, D-2300 Kiel 1, Germany

\section{Ernst Niebur} \\ Computation and Neural Systems 216-76, Caltech, Pasadena, CA 91125 USA
}

We study the dynamics of completely connected populations of refractory integrate-and-fire neurons in the presence of noise. Solving the master equation based on a mean-field approach, and by computer simulations, we find sustained states of activity that correspond to fixed points and show that for the same value of external input, the system has one or two attractors. The dynamic behavior of the population under the influence of external input and noise manifests hysteresis effects that might have a functional role for memory. The temporal dynamics at higher temporal resolution, finer than the transmission delay times and the refractory period, are characterized by synchronized activity of subpopulations. The global activity of the population shows aperiodic oscillations analogous to experimentally found field potentials.

\section{Introduction}

Most artificial neural networks are based on binary McCulloch-Pitts neurons that have no intrinsic temporal characteristics. As opposed to these simplified units, real neurons perform temporal integration over their inputs with some specific decay constant and have refractory periods. Model neurons satisfying these constraints are often called integrate-andfire neurons. Although the dynamic behavior of a single such neuron is straightforward, their population dynamics are highly complex. Recently, several studies have shown that under specific assumptions analytical results characterizing such populations of neurons can be obtained. For example, Amit and Tsodyks (1992) have shown that on a longer time scale the dynamic behavior of integrate-and-fire neurons can be averaged 
out and characterized by continuous variables (firing rates or currents). However, this transformation is based on the assumption that there is no synchronicity among inputs and once made, the question of dynamics at higher temporal resolution cannot be addressed. Gerstner and Van Hemmen (1992) proposed a stochastic model for spiking neurons that incorporates refractory periods and transmission delays, but which disregards membrane decay properties. The issue of temporal modulations has been investigated by Mirollo and Strogatz (1990), who showed that a population of integrate-and-fire neurons in a strong external field (receiving a strong external input and acting as pulse oscillators) will phaselock and reach synchronicity if they have excitatory all-to-all connections. Van Vreeswijk and Abbott (1992) analyzed the behavior of completely connected populations of excitatory neurons and found that even in the absence of external input, such populations of neurons can exhibit sustained activity due to self-interaction. In particular, they showed that, in the absence of noise, the population may lock in one of several patterns of cyclic activity.

The purpose of this work is to further analyze the dynamic characteristics of such populations in the presence of noise and small external inputs, and to find the specific way that a population responds to different inputs. In the first part we develop a discrete-time mean-field model, using a master equation that takes explicitly into account the stochastic behavior due to noise. Using the master equation and numerical simulations we show that for some values of the external field the population is dominated by two attractors, a self-sustained activity state and a silent state. These states are fixed points of the dynamics, and we discuss their basins of attraction. We also show that the network exhibits hysteresis and thus can function as a memory system that reacts to external inputs in a different way than systems consisting of McCulloch-Pitts neurons. In the last section, we study the temporal dynamics at a finer temporal resolution. Taking explicitly into account axonal and synaptic delays and refractory periods, we obtain synchronized activity of subpopulations and aperiodic oscillations of the whole population's activity.

\section{The Model}

Consider a fully connected population of $N$ excitatory neurons, each one characterized by a continuous variable $z_{i}$ that represents the cell's potential $(1 \leq i \leq N)$. Each neuron integrates over its inputs, and once it reaches a threshold (chosen without loss of generality to be 1 ), the neuron fires, sends its output to the other neurons in the population, and resets its potential to zero. For simplicity, we will use a synchronous updating rule where each iteration step corresponds to a typical time for the spike dynamics (including the refractory period) and transmission delays of 
about 2-3 msec. Thus for neuron $i$,

$$
z_{i}(t+1)=\left\{\lambda z_{i}(t)+\frac{J}{N} \sum_{j=1}^{N} \Theta\left[z_{j}(t)-1\right]+\xi_{i}+h\right\} \Theta\left[1-z_{i}(t)\right]
$$

where $\lambda$ is a decay constant $(0 \leq \lambda \leq 1), J>0$ is the coupling constant, $\Theta$ is the unit step function, and the external input is characterized by a mean value $h$ and a gaussian noise term $\xi_{i}$ (of zero mean and standard deviation $\sigma$ ). The first step function adds the contributions from other cells that are firing at time $t$, and the second step function enforces refractoriness (a neuron cannot fire at two consecutive time steps).

In order to test the validity of our synchronous discretization scheme, we have to test whether its dynamic properties depend upon the length of the iteration steps (in the limit of infinitesimal time steps, our scheme should reduce to a continuous system of differential equations representing currents in the cells). As it stands, equation 2.1 implies that both the delay time and the refractory period are equal to the discretization steps. Thus in order to increase the temporal resolution (without changing the actual values of the biological parameters, i.e., refractoriness and delay time), equation 2.1 should be modified, so that the refractory period and the delay time extend over more than one (now smaller) time bin. We shall show in the last section that the results obtained from equation 2.1 (at low time resolution) are compatible with those obtained from the modified delayed equation. However, at the higher resolution new phenomena are revealed.

As shown in Van Vreeswijk and Abbott (1992) for zero external input and noise $\left(h=\xi_{i}=0\right)$, the population may either remain silent or segregate into a cyclic pattern of activity of length $M$. At time $t$ a subgroup of $n^{t}$ cells fire and subsequently, at time $t+1$, a different subgroup of $n^{t+1}$ cells will fire etc., satisfying $\sum_{t}^{t+M} n^{t}=N$. For $\lambda=1$ the length of the cycle $M$ is limited (Van Vreeswijk and Abbott 1992) by:

$$
\frac{J N}{J N-1}<M<\frac{2 J N}{N J-1}
$$

Although the length of the cycle is limited by this equation, this does not determine the sequence of activations $n^{t}, n^{t+1}, \ldots, n^{t+M}$, which depends on the initial conditions $z_{i}(0)$. As we shall show in the next section, under the influence of noise the temporal behavior is asymptotically characterized by homogeneous sequences [i.e., $n(t)=n(t+1) \ldots=n(t+M)$ ]. Thus the system converges to one of two possible fixed points, a homogeneous sequence of activations or the silent state. This situation is somewhat analogous to a ferromagnetic system.

\section{Master Equation}

The stochastic system in equation 2.1 is completely characterized by the full probability distribution $\mathcal{P}^{t}\left(z_{1}, z_{2}, \ldots, z_{N}\right)$. Since our system is fully 
connected, a mean-field approach yields a lower dimensional-probability distribution $P^{t}(z)$,

$$
P^{\prime}(z)=\frac{1}{N} \sum_{i} \int \delta\left(z-z_{i}\right) \mathcal{P}^{\prime}\left(z_{1} \ldots, z_{N}\right) d z_{1} \ldots d z_{N}=\frac{1}{N} \sum_{i} P_{i}^{\prime}(z)
$$

where $P_{i}^{\prime}(z)$ is the probability that neuron $i$ will have the synaptic potential $z$ at time $t$, and $P^{t}(z)$ is the mean-field average probability of having a postsynaptic potential $z$ in the system at time $t$. The time dependence of $P^{t}(z)$ is obtained from equation 2.1:

$$
P^{t+1}(z)=m^{t} \delta(z)+\int_{-\infty}^{1} d z^{\prime}<\delta\left[z-\left(\lambda z^{\prime}+J m^{t}+h+\xi\right)\right]>P^{\prime}\left(z^{\prime}\right)
$$

where $m^{t}$, the normalized fraction of active cells at time $t$ is related to $P^{t}(z)$ via $m^{\prime}=\int_{1}^{\infty} P^{t}(z) d z$, and $<>$ denotes the average over the noise probability distribution that is chosen to be gaussian:

$$
P_{\text {noise }}(\xi)=f(\xi)=\frac{1}{\sigma \sqrt{2 \pi}} e^{-\xi^{2} / 2 \sigma^{2}}
$$

Inserting this into equation 3.1 we obtain,

$$
P^{t+1}(z)=m^{t} \delta(z)+\int_{-\infty}^{1} d z^{\prime} f\left(z-\lambda z^{\prime}-J m^{t}-h\right) P^{t}\left(z^{\prime}\right)
$$

from which $m^{t+1}$ can be calculated

$$
m^{t+1}=\int_{1}^{\infty} d z P^{t+1}(z)=\int_{-\infty}^{1} d z^{\prime} \int_{1}^{\infty} d z f\left(z-\lambda z^{\prime}-J m^{t}-h\right) P^{t}\left(z^{\prime}\right)
$$

3.1 Strong Decay. For $\lambda=0$, the integral in equation 3.4 factorizes and we obtain a nonlinear one-dimensional map:

$$
m^{t+1}=\left(1-m^{t}\right) \int_{1-J m^{t}-h}^{\infty} d \xi f(\xi)
$$

Approximating the gaussian by $f(\xi)=\left\{2 T \cosh ^{2}(\xi / T)\right\}^{-1}$ with $T:=$ $\sigma \sqrt{\pi / 2}$, we obtain

$$
m^{t+1}=\frac{1-m^{t}}{2}\left\{1+\tanh \frac{J m^{t}+h-1}{T}\right\}
$$

The dynamic behavior of $m^{t}$ is governed by the parameters $J, h$, and $T$. In Figure 1a-c (solid lines) we plot the term on the right-hand side of equation 3.6 together with the identity function, for $T=0.2, J=1.5$, and different values of the external field $h$. For high external field $h=0.8$ (Fig. 1a), the map has a single fixed point characterized by an activation value close to 0.5 , which implies that for such high input the population segregates in two subgroups that fire consecutively ( 0.5 is the maximal 
(a)

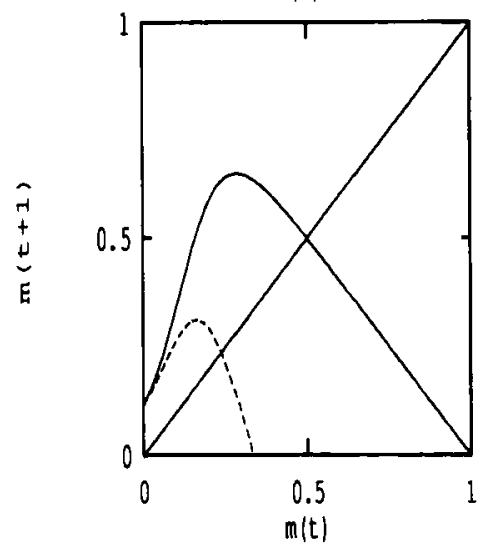

(c)

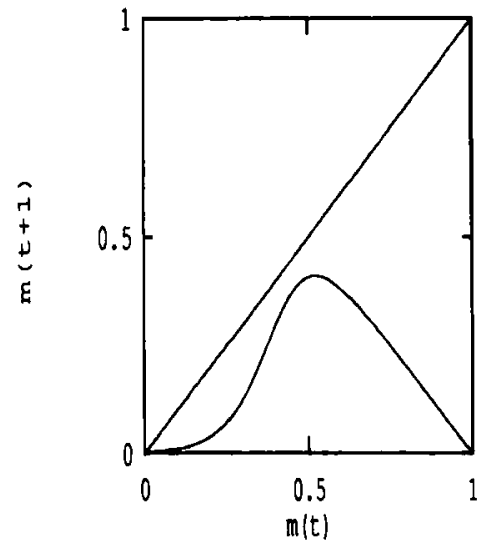

(b)

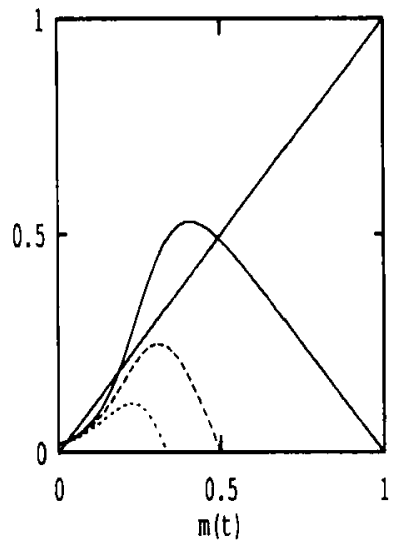

(d)

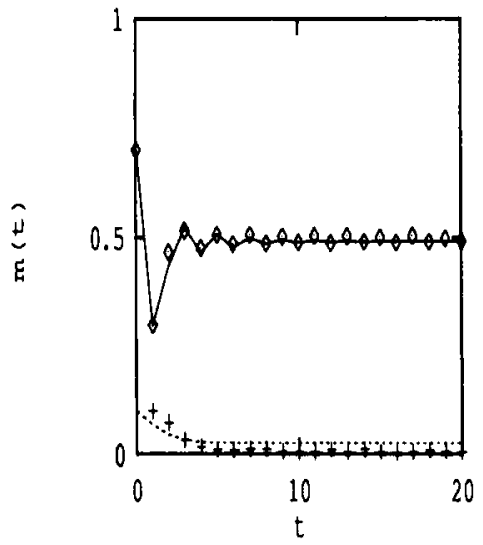

Figure 1: The one-dimensional map (equations 3.4 and 4.4) for $J=1.5, T=0.2$ and different external fields: (a) $h=0.8$, equation 3.4 solid line, and equation 4.4 with $n_{d}=n_{r}=3$ dashed line; (b) $h=0.6$, equation 3.4 solid line and equation 4.4 with $n_{d}=n_{r}=2$ long dashed line, $n_{d}=n_{r}=3$ short dashed line; (c) $h=0.4$, equation 3.4; (d) trajectories beginning from different initial conditions $\left(m^{0}=0.1\right.$ or $m^{0}=0.7$ ) for parameters corresponding to (b). Lines, solutions of equation 3.4; symbols, simulation with a population of $N=1000$ neurons for the two initial conditions shown. 
firing rate due to the refractoriness). The fixed point is stable and thus the temporal sequence of activations will manifest damped oscillations toward the stable fixed point. For intermediate values of the external field, the system has two stable fixed points and an unstable one (the intermediate one). Therefore, for such external fields the system has two attractors and can function as a memory system. The domains of attraction of each fixed point are unlike those in a McCulloch-Pitts system; the initial states that are attracted toward the high $m$ value have intermediate $m(0)$, while the very low and very high values $m(0)<0.14$ or $m(0)>0.8$ lead to the decay of the activation. This is illustrated in Figure $1 \mathrm{~d}$, where we display the time sequence obtained from two different initial conditions using the one-dimensional map (lines) and a simulation with $N=1000$ (symbols). A further decrease of the external field leads to the situation displayed in Figure $1 \mathrm{c}$, where the activity is always decaying.

For $0<\lambda \ll 1$ a two-dimensional map can be obtained, using a first order Taylor expansion of equations 3.3 and 3.4. For moderate values of $\lambda$ (e.g., $\lambda=0.4)$, the system behaves qualitatively similar to a system with $\lambda=0$, that is, it has a single attractor with high $m$ for a large external field, two attractors for intermediate external fields, and again a single attractor (with small $m$ ) for small external fields. Due to the finite value of $\lambda$, the transition points between these regimes occur at smaller field values than for $\lambda=0$ (e.g., for $\lambda=0.4$, the system has 2 attractors already for $h=0.4$; data not shown).

3.2 Weak Decay. We have numerically solved the master equation for an initial distribution $P^{0}(z)$ corresponding to a fraction of active neurons $m^{0}$, uniformly distributed otherwise. For $\lambda=1$, we found that the system always reaches a fixed point corresponding to a homogeneous sequence $m^{t}=1 / M$ (where $M$ is compatible with equation 2.2), even for vanishing external input. This is illustrated in Figure $2 \mathrm{a}$ where beginning with a very high initial activation (which would lead to decay in the absence of noise), the system gradually recovers due to small gaussian noise (with $\sigma$ corresponding to $T=0.05$ in equation 3.6). This phenomenon is explained by the fact that noise causes diffusion of the probability distribution $P(z)$, whose tail eventually reaches the threshold and turns the system on. Also due to noise, the system equalizes the size of the subgroups. Figure $2 \mathrm{~b}$ shows the multimodal probability distribution (with four components corresponding to the $M=4$ subgroups) reached after 20 iterations. The same stationary distribution is reached from all initial conditions.

For $\lambda<1$, the noise-induced diffusion is balanced by the decay, and the system is not able to self-activate for all initial conditions. As in the small $\lambda$ case, the system will reach one of two attractors, depending on the initial conditions. In Figure $2 c$ we display the time sequence obtained for $\lambda=0.98$, beginning with initial condition $m^{0}=0.28$, and 
(a)

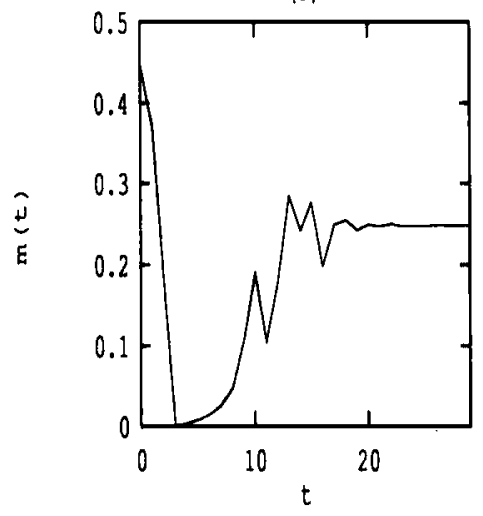

(c)

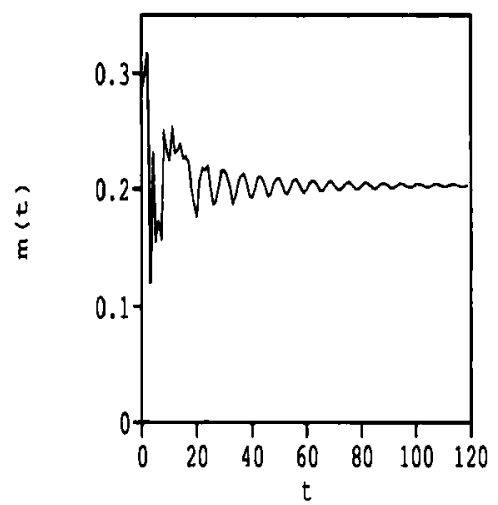

(b)

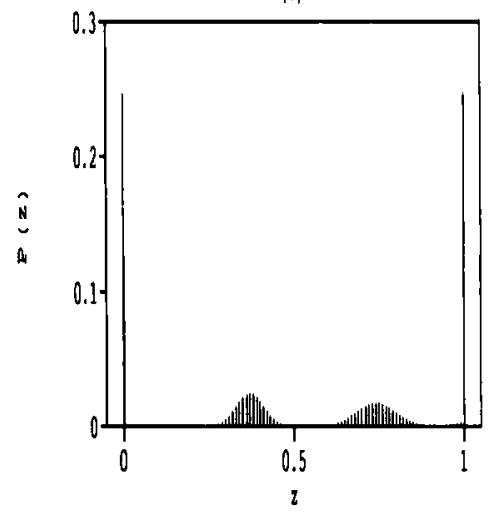

(d)

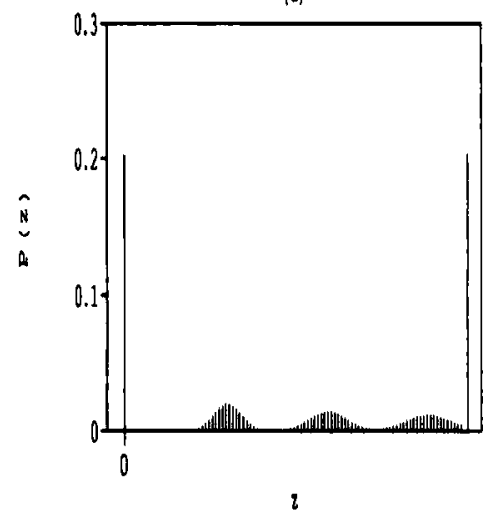

Figure 2: Solution $m^{t}$ of the master equation, equation 3.4 , for $P^{0}(z)=m^{0} \delta(z-$ $1)+\left(1-m^{0}\right)[\theta(z)-\theta(z-1)], J=1.5, T=0.05, h=0$. (a) $m^{t}$ for $\lambda=1, m^{0}=0.44$, (b) $p^{t}(z)$ for $t=30$. (c) $m^{t}$ for $\lambda=0.98, m^{0}=0.28$. (d) Probability distribution after $t=120$ iterations, parameters as in (b). In both cases, the system reaches a fixed point of self-sustained activity.

$P^{0}(z)$ uniform otherwise. We observe damped oscillations that reach a fixed point of $m=0.2$, implying segregation into five equal subgroups. The corresponding multimodal probability distribution $P(z)$ is displayed in Figure 2d. Noise tends to homogenize the initially nonhomogeneous sequence of activation by transporting probability from the larger to the smaller subgroups. Begining with a "too high" activation $m^{0}=0.44$ and 

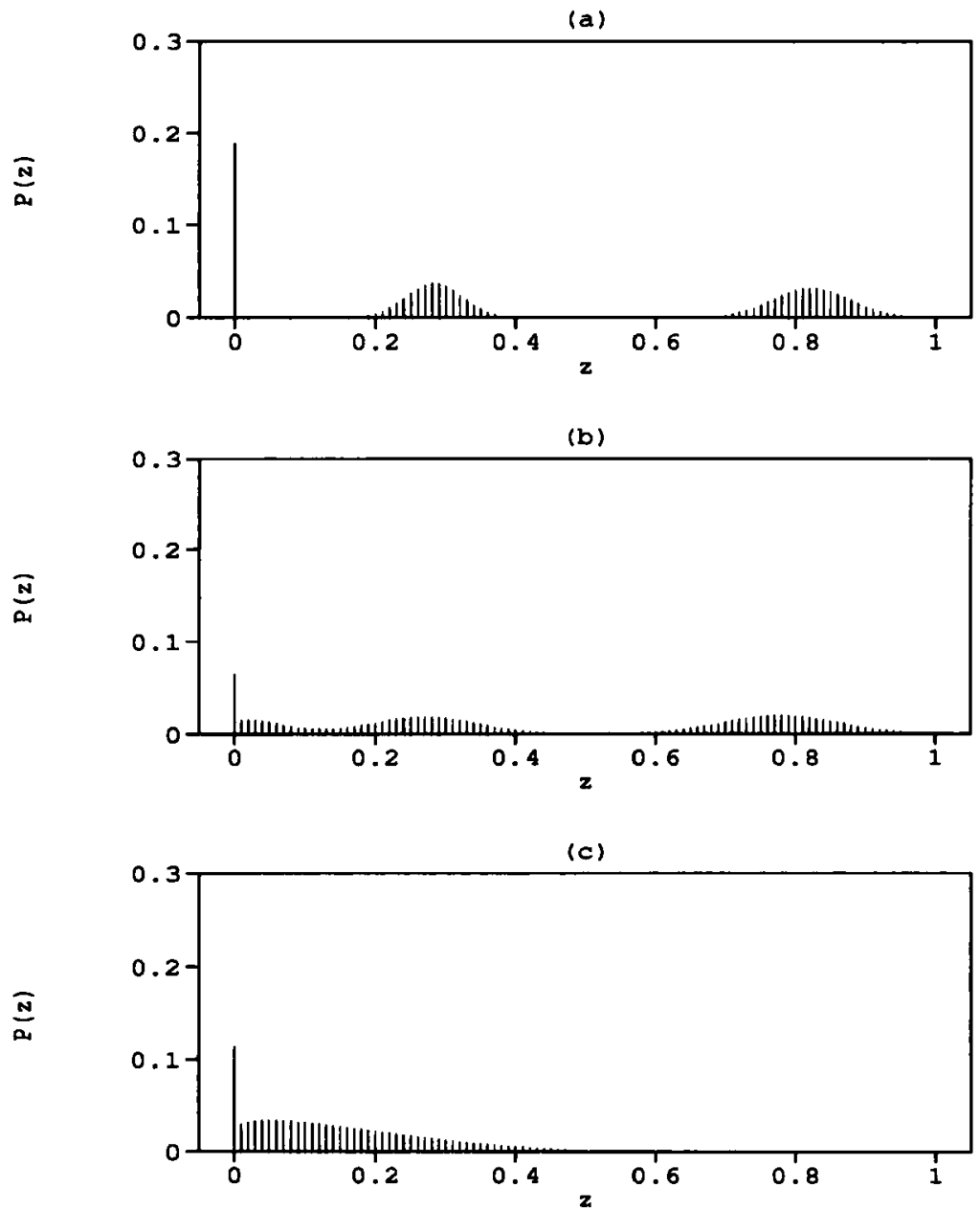

Figure 3: $P^{t}(z)$, same parameters as in Figure 2, beginning with a high initial activation $m^{0}=0.44, p^{0}(z)=m^{0} \delta(z-1)+\left(1-m^{0}\right)[\theta(z)-\theta(z-1)]$. (a) $t=3$, (b) $t=9$, (c) $t=120$ iterations. The probability shrinks due to the decay, although, of course, $\int d z P^{t}(z)=1$ for all $t$. After 5 iterations, $m^{t} \approx 0$.

$P^{0}(z)$ uniform otherwise, the activation decays to zero. In Figure 3 we display the probability distribution $P^{t}(z)$ at $t=3, t=9$, and $t=120$. We observe that in spite of the diffusion, $P^{t}(z)$ shrinks and the populations remain inactive. 
The influence of the noise on the system dynamics can be summarized as follows: For very low noise (small $T$ ), the system reaches a limit cycle in which the system segregates into subpopulations of unequal sizes in each of which all neurons fire synchronously. The length of the limit cycle depends on the initial conditions. When the noise exceeds a certain value, a transition toward a fix-point representing segregation into equal subpopulations occurs, and the dependence on initial conditions reduces to the choice between 2 attractors (the silent and the active state). The noise value at which the transition occurs depends on the number of subpopulations in the cycle; for $J$ larger than, but near 1 , cycles of long length are obtained, as shown in Figure 4a. In this case the different subpopulations are separated by small potential differences, and very low noise will transport neurons between the different subpopulations and contribute to their homogenization (Fig. 4b). As $J$ increases, the cycle length (and hence the number of subpopulations) decreases while the potential differences between them increase. Thus a higher noise level is required in order to transport neurons among the populations. A systematic simulation study of the $J$ dependency shows the following:

- For $J=1.9, \lambda=1$, a cycle of order 3 is obtained in the absence of noise, and the temperature required for inducing the transition is $T=0.07$.

- For $J=1.5, \lambda=1$, a cycle of order 4 is obtained, and the temperature required for inducing the transition was $T=0.04$.

- For $J=1.1$ a cycle of length 14 was obtained, and a noise characterized by $T=0.01$ was enough to induce the transition (Fig. $4 \mathrm{~b}$ ).

3.3 Inputs and Hysteresis. There are two ways by which a neural population can receive external inputs. The first one is via the initial state $m^{0}$ (i.e., a subgroup of the population is turned on), and the second one is by sustained external input (in form of postsynaptic potentials), that is modeled as an external field $h$. For $h=0$, we have shown that the system has two attractors. This leads to the hysteresis phenomenon illustrated in Figure 5, where we show the average activation of the population, as we increase (or decrease) adiabatically the external field. We observe that for $h=0$ the activation may have two values depending on history. Therefore, absence of excitation (or presence of small inhibition) will leave the population inactive. However, once positive excitation has reached the assembly causing it to fire, the population "remembers" and continues self-sustained firing even after the external input no longer reaches the population. 
(a)

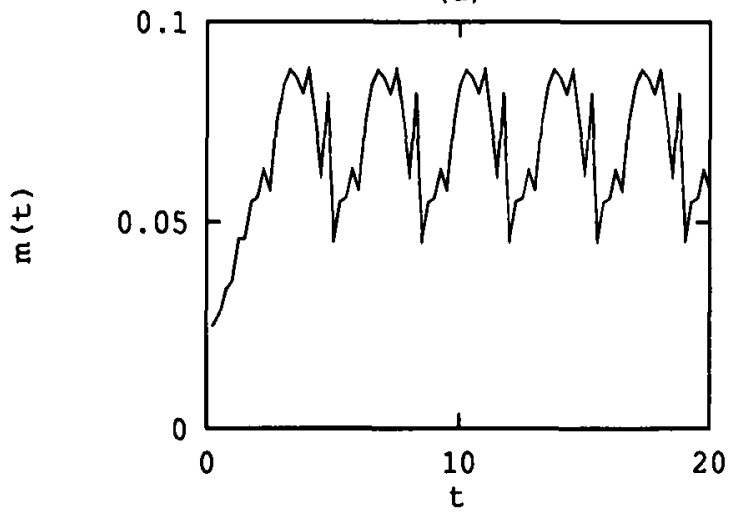

(b)

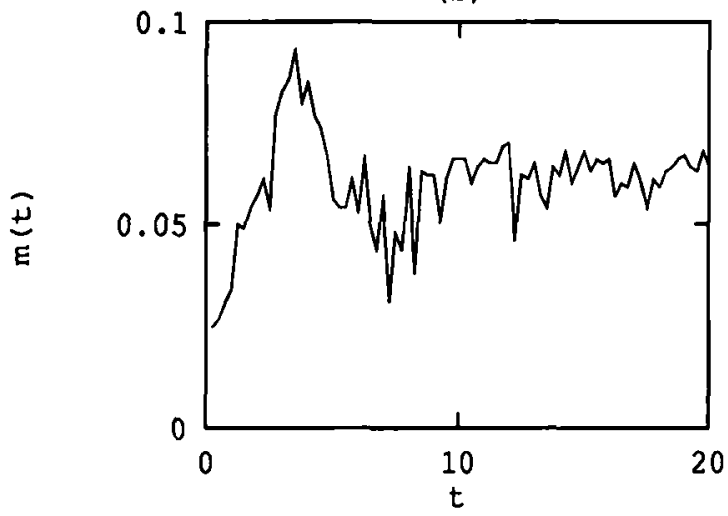

Figure 4: Long cycles for $J=1.1$. (a) Without noise $(T=0)$, a cycle of length 14 is obtained, (b) a small level of noise $(T=0.01)$ leads to stochastic fluctuations around a homogeneous steady state.

4 Dynamics at Higher Time Resolution; Synchronicity and Field Potentials

As we have already mentioned, equation 2.1 has to be modified in order to investigate the population dynamics on a time scale characterized by a finer discretization. This is accomplished by considering a bin size that equals a fraction, say $1 / n$, of the characteristic delay time and refractory 
(a)

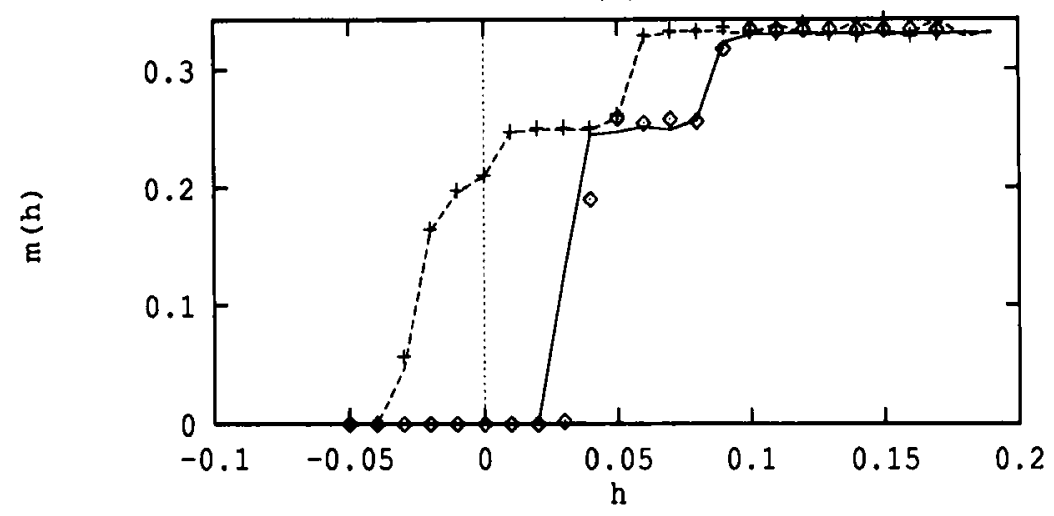

(b)

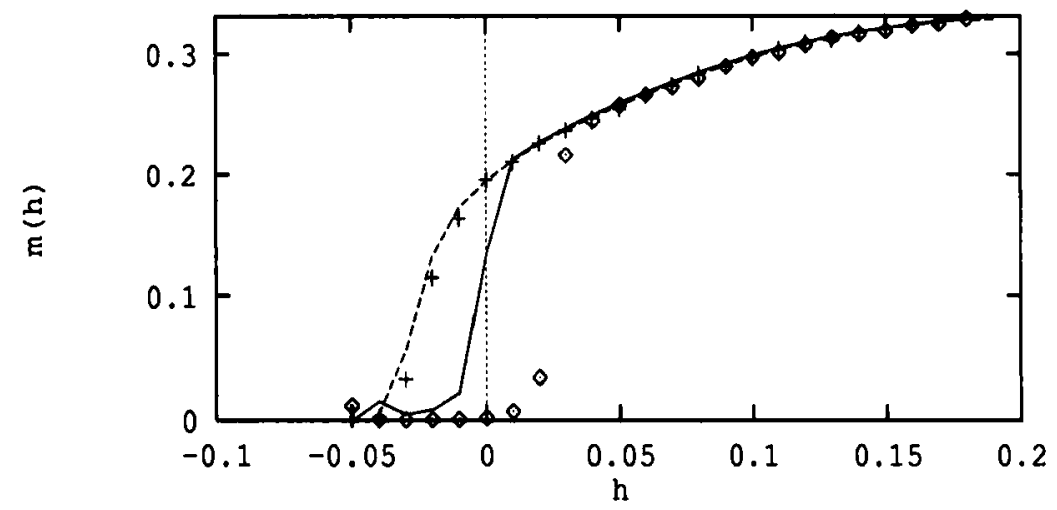

Figure 5: Hysteresis. The average $m^{t}$ is plotted as a function of the adiabatic field $h$. (The field was increased by 0.01 every 20 iterations, and the average activation $m$ was plotted.) Lines represent numerical solutions of the master equation 3.4; symbols represent simulations of a population of $N=1000$ cells. (a) Low noise $(T=0.05)$. Note the jumps caused by quantal transitions between cyclic patterns of length $M=4$ and $M=3$. (b) Same for higher noise level $T=0.2$. Parameters $\lambda=0.98, J=1.5$ for (a) and (b).

period. We assume in the following that all pairs of cells are characterized by a single communication delay time (composed of axonal and synaptic delay), but we shall discuss the case when the delay is different from the refractory time. Each neuron integrates over its inputs, and once it 
reaches the threshold, the neuron fires, sending its output to the other neurons in the population where it will arrive after a delay of length $n_{d}$. After emitting the spike the neuron is reset to zero, and remains refractory for $n_{r}$ time steps. The modified equation is

$$
\begin{aligned}
z_{i}(t+1)= & \left\{\lambda z_{i}(t)+J / N \sum_{j=1}^{N} \Theta\left[z_{j}\left(t-n_{d}\right)-1\right]+\xi_{i}+h\right\} \\
& \times \Theta\left[1-z_{i}(t)\right] \ldots \Theta\left[1-z_{i}\left(t-n_{r}\right)\right]
\end{aligned}
$$

where $n_{d}$ and $n_{r}$ stand for the delay time and the refractory period in units of the discretization, respectively. Increasing the temporal resolution corresponds to using larger values for these variables. We notice that the transformation from equation 2.1 to equation 4.1 implies a corresponding scaling of the decay constant $\lambda=\lambda^{1 / n_{d}}$, and of the temperature $T=T / \sqrt{n}_{d}$.

The behavior of the neural population in the presence of noise can be characterized by a Markov process with memory of order $n_{d}$,

$$
\begin{aligned}
P^{t+1}(z)= & \left(m^{t}+m^{t-1} \ldots m^{t-n_{r}}\right) \delta(z) \\
& +\int_{-\infty}^{1} d z^{\prime} \ldots d z^{\left(n_{d}\right)} f\left(z-\lambda z^{\prime}-J m^{t-n_{d}}-h\right) \\
& \times P\left[z^{\prime}(t), z^{\prime \prime}(t-1) \ldots z^{\left(n_{d}\right)}\left(t-n_{d}\right)\right]
\end{aligned}
$$

where the probability $P^{t}\left(z^{\prime}\right)$ is replaced by $P\left(z^{\prime}, z^{\prime \prime}, \ldots\right)$, the probability that a neuron had value $z^{\prime}$ at time $t, z^{\prime \prime}$ at time $t-1$, and so on. We should notice that this probability is not multiplicative due to temporal correlations, for example, for the case $n_{d}=2$,

$$
\begin{aligned}
\operatorname{Prob}\left[z^{\prime}(t)<1, z^{\prime \prime}(t-1)<1\right] & =1-m(t)-m(t-1) \\
& \neq[1-m(t)][1-m(t-1)]
\end{aligned}
$$

From equation 4.2, a map analogous to equation 3.6 can be obtained for $\lambda=0$ :

$$
m^{t+1}=\frac{\left(1-m^{t}-m^{t-1}-\cdots-m^{t-n_{r}}\right)}{2}\left\{1+\tanh \frac{J m^{t-n_{d}}+h-1}{T}\right\}
$$

The solutions of this delay equation are more complex than the analogous solutions of equation 3.6. However, special solutions that also satisfy equation 3.6 can be obtained easily. Consider the following 2 cases:

- The activation for each subpopulation is spread homogeneously at the subrefractory time resolution, that is, $m^{t}=m^{t-1}=\cdots=m^{i-n_{r}}$. 
In this case, and for $n_{d}=n_{r}$, equation 4.3 transforms into:

$$
m^{i+1}=\frac{1-n_{r} m^{t}}{2}\left\{1+\tanh \frac{I m^{t}+h-1}{T}\right\}
$$

where the time bin equals the delay and the refractory period.

- Perfect synchronization of subpopulations, that is, $m^{t} \neq 0, m^{t-1}=$ $\cdots=m^{t-n_{d}}=0$ repeating cyclically modulo $n_{d}$. Such periodic solutions can be studied easily, since they also satisfy equation 3.6.

We have shown that for $\lambda=0, J=1.5, T=0.2$ the population may converge to an attractor of high activity for high field values (e.g., $h=0.8$, Fig. 1a, solid line), to the silent state for low field values ( $h=0.4$, Fig. 1c, solid line), or to two attractors, for intermediate field values ( $h=0.6$, Fig. 1b, solid line), depending on the initial activation. When the high resolution dynamics are taken into account (equation 4.3), the following characteristics are revealed:

For $h=0.8$ both the homogeneous case (equation 4.4 and Fig. 1a, dashed line) and the synchronized case (Fig. 1a, solid line) have nontrivial solutions. For intermediate field values $h=0.6$, only the synchronized solution is nontrivial; Fig. $1 \mathrm{~b}$ (dashed lines) displays the map in equation 4.4 showing that for $n_{d}=n_{r} \geq 2$, the only solution is the silent state. Numerical iteration of the map, equation 4.3 , and simulations confirm that for high external field either a homogeneous or a modulated solution is obtained (depending on initial conditions), while for intermediate fields (that are essential for memory preservation) only the synchronized solutions remain.

Finally, we present simulation results that display the high resolution dynamics, equation 4.1, for weak decay ( $\lambda$ close to one). In Figure 6a, the system parameters were chosen identical to those used in Figure $2 c$ (subject to the scaling of $\lambda$ and $T$ ) and $n_{d}=n_{r}=4$. We see that the population's activity manifests rapid oscillations (of period equal to the delay time), with amplitude $m=0.2$, reflecting a segregation into five populations (as in Fig. 2c). Figure 6b displays a close-up into Figure 6a showing that inside each such population, there is a relatively high but variable synchronicity that leads to slower and irregular waves superimposed on rapid oscillations (the periodicity of the signal in this synchronized state is imposed by the transmission delay).

If the delay time is larger than the refractory period, a different behavior is obtained. In Figure $6 \mathrm{c}$ we illustrate the population dynamics for the same parameters as in Figure 6a, except that $n_{d}=5$, while $n_{r}=4$ as before. We observe that the activity oscillates with the period of the delay time, but the amplitude is increased to $m=0.5$, implying segregation into 2 subpopulations. The reason for this change is that unlike in the previous case $\left(n_{d} \leq n_{r}\right)$ no activation is lost, that is, cells can send activation to themselves, since after the delay they are not refractory any 

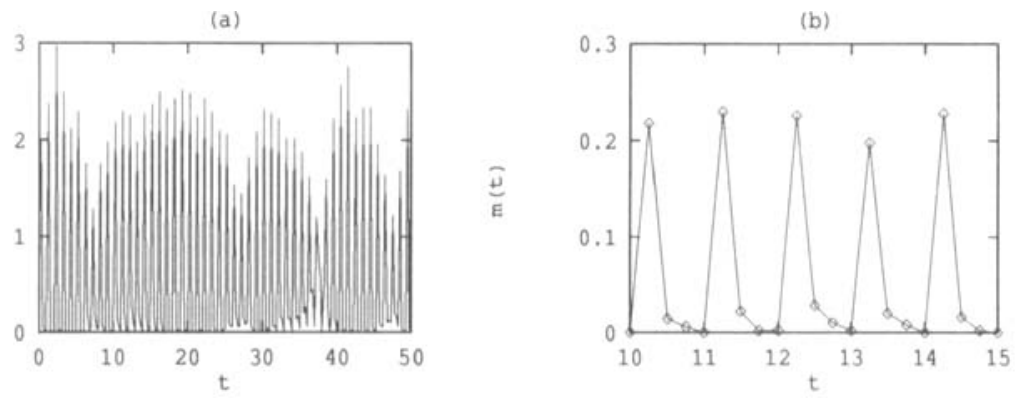

(c)
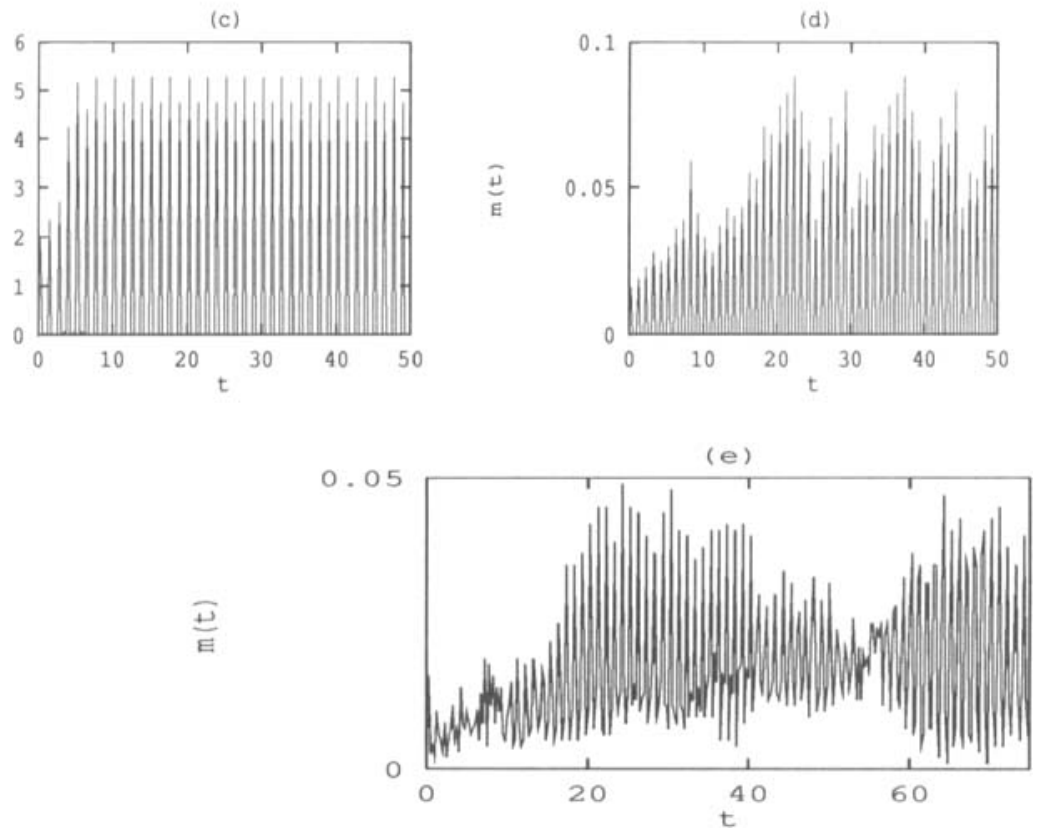

Figure 6: Simulations with higher temporal resolution (equation 4.1), $N=1000$ neurons. (a) Parameters as in Figure 2b, except $n_{d}=n_{r}=4$; (b) close-up showing that the periodicity is determined by the transmission delay; (c) $n_{d}=5$, $n_{r}=4$; (d) parameters as in Figure 4a, $n_{d}=n_{r}=4, T=0$; (e) same with noise $T=0.01$.

more. In such a case, complete synchronization of the population is obtained for higher values of $J$ in which all neurons fire with the period of the delay.

In Figure 6d, we display the high resolution simulation $\left(n_{d}=n_{r}=4\right)$, 
corresponding to small $J$ values leading to long cycles (parameters as in Fig. 4). In the absence of noise, a periodic solution (periodicity $15 n_{d}$ ) analogous to Figure $4 a$ is obtained. Adding a noise factor equivalent to the one used in Figure $4 \mathrm{~b}$, we observe that the activity manifests irregular oscillations (Fig. 6e) whose maximal amplitude is $m=0.05$, implying segregation into approximately 20 subpopulations. The synchronization of each subpopulation varies stochastically, and thus the total activity is characterized by aperiodic spindles of high oscillations. Such spindles are highly suggestive of experimental field potentials or EEG recordings. Since these are also considered to reflect a global activity in some neural population, as it is the case in our model, we think that our simple model provides a simple explanation of possible mechanisms underlying these phenomena.

\section{Discussion}

Most neural network models are built of simplified units that do not take into account inherent properties of real neurons, such as refractory period and integration time. Such properties are essential for any computational process dealing with the temporal structure of neural activity. For example, in the absence of an integration constant, small jitter in the transmission of signals would wash out synchronicity effects. Thus networks based on refractory integrate-and-fire neurons are among the simplest models that may explain how the brain processes temporally structured information. However, despite the widespread use of integrate-and-fire neurons as models for single cells, only recently systematic studies of the population dynamics of such networks have been carried out (Amit and Tsodyks 1992; Ahlstrom and Levinsen 1992; Kuramoto 1992; Van Vreeswijk and Abbott 1992).

In the present work, we have studied the behavior of large, completely connected networks of refractory integrate-and-fire neurons in the presence of homogeneous input and noise. Two results seem particularly noteworthy. One is the finding that at the time resolution of the synaptic delay, the presence of noise will always lead to a segregation of the system in neuronal groups of equal size. Thus on time scales larger than the transmission delay, our results support an interpretation of steady state firing rate as in Amit and Tsodyks (1992). However, at a finer time resolution, firing may be highly synchronized and temporally modulated with the periodicity of the delay time, as in the model proposed in Gerstner and Van Hemmen (1992).

The second important result is the observation of hysteresis in the system. It is tempting to speculate that this is a mechanism employed for the neural implementation of short-term memory. The idea of memory being implemented in the brain by reverberating activity dates back at least to Caianiello and has received new vigor by recent observations showing 
that the activation of short-term memory is correlated with increased neural activity in neocortex (Miyashita and Chang 1988; Fuster 1990). Our results indicate that self-excitatory networks of integrate-and-fire neurons are able to manifest a basic form of this behavior, especially when they operate in the synchronized mode. As we have shown, when the system is in its memory mode, characterized by two attractors, only the synchronous (and not the homogeneously spread-out solution) is nontrivial. The main reason for this is that synchronized activity is more effective in activating other cells than randomly spread activity. This may also shed light on the widely argued discussion about the role of cortical oscillations in information processing. According to our model, aperiodic oscillations and synchronicity are emergent properties of neural populations with recurrent excitation, and contribute to its memory function.

The dynamic properties of such systems are different from the memory systems based on McCulloch-Pitts neurons. For example, when transmission delays are equal or smaller than the refractory period, a transient external input is more effective in inducing sustained activity in populations of integrate-and-fire neurons if the input is at an intermediate value, rather than at a relatively high value. The significance of such dynamic characteristics to the actual physiological process underlying short-term memory, and the use of integrate-and-fire neurons for modeling physiological time dependent processes should be the subject of further research.

\section{Acknowledgments}

EN is supported by the Office of Naval Research and wishes to thank HGS and the Institute for Theoretical Physics of the University of Kiel for their hospitality. HGS thanks Christof Koch for his kind hospitality during his stay at Caltech. MU is supported by a Bantrell fellowship. This work was supported by NATO Grant 911034 COP.

\section{References}

Ahlstrom, P., and Levinsen, M. T. 1992. Phase-locking structure of integrateand-fire models with threshold modulation. Phys. Lett. A 128(3-4), 187-192.

Amit, D. J., and Tsodyks, M. V. 1992. Quantitative study of attractor neural network retrieving at low spike rates. Network 2(3), 259-273.

Fuster, J. M. 1990. Inferotemporal units in selective visual attention and shortterm memory. J. Neurophysiol. 64, 681-697.

Gerstner, W., and Van Hemmen, J. L. 1992. Associative memory in a network of 'spiking' neurons. Network 3, 139-164.

Kuramoto, Y. 1992. Collective synchronization of pulse-coupled oscillators and excitable unit. Physica D 50(1), 15-30. 
Mirollo, R. E., and Strogatz, S. H. 1990. Synchronization of pulse-coupled biological oscillators. SIAM J. Appl. Math. 50(6), 1645-1662.

Miyashita, Y., and Chang, H. S. 1988. Neural correlate of pictorial short term memory in primate temporal cortex. Nature (London) 331, 68-70.

Van Vreeswijk, C., and Abbott, L. F. 1992. Self-sustained firing in populations of integrate and fire neurons. SIAM J. Appl. Math.

Received 14 September 1992; accepted 17 November 1992. 